\title{
Modified PTS-based PAPR reduction for ACO-OFDM in visible light communications
}

\author{
TAN Jiandong, WANG Qi \& WANG Zhaocheng* \\ Tsinghua National Laboratory for Information Science and Technology (TNList), \\ Department of Electronic Engineering, Tsinghua University, Beijing 100084, China \\ Received ; accepted
}

Citation Tan J D, Wang Q, Wang Z C. Modified PTS-based PAPR reduction for ACO-OFDM in visible light

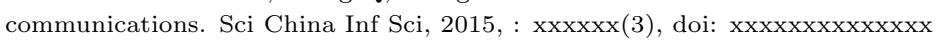

\section{Dear editor,}

Visible light communication (VLC) has raised great interests since it supplements the unlicensed spectrum resource for ultra-high data rate transmission [1]. In order to resist time dispersion and guarantee the real-valued and non-negative constraint in VLC channel, DC-biased optical orthogonal frequency-division multiplexing (DCO-OFDM) and asymmetrically clipped optical OFDM (ACO-OFDM) techniques have been utilized in VLC systems [2]. Unlike DCO-OFDM which requires high DC bias [3], ACO-OFDM enjoys an advantage of high power efficiency and wide light emitting diodes (LED) dynamic region.

The inherent peak-to-average power ratio (PAPR) issue of VLC OFDM leads to severe LED nonlinear distortion, which deteriorates the bit error rate (BER) performance [1]. Several methods reported in [4-7] have been investigated to reduce PAPR and mitigate LED nonlinear distortion of OFDM-based VLC systems. However, the costs such as data rate and performance loss induced by the above algorithms could not be neglected.

As an effective method for PAPR reduction, partial transmit sequence (PTS) technique has an advantage of negligible performance degradation and no power increase. However, side information is required at the receiver, which results in data throughput loss [8]. In this letter, we propose a modified PTS-based PAPR reduction scheme for ACO-OFDM in VLC systems by exploiting the unoccupied subcarriers to transmit side information. A novel transmitter is presented to apply the PTS technique in ACO-OFDM VLC system without data throughput loss and an original power allocation method is also proposed to mitigate the power efficiency loss induced by the side information signals. Simulation results demonstrate that the proposed scheme achieves a significant PAPR reduction performance without data throughput loss as well as an improved BER performance when LED nonlinearity exists.

Methodology. At the transmitter, the quadrature amplitude modulation (QAM) symbols $\mathbf{X}_{\mathrm{ACO}}$ of $N$-point ACO-OFDM block is divided into $V$ disjoint clusters $\left\{\mathbf{X}_{\mathrm{ACO}}^{(i)} \mid i=0,1, \ldots, V-1\right\}$ by adjacent partitioning scheme. By taking $V$ clusters of inverse fast Fourier transform (IFFT) operation, $\mathbf{X}_{\mathrm{ACO}}^{(i)}$ is converted to the time-domain cluster vector $\mathbf{x}_{\mathrm{ACO}}^{(i)}$, which is denoted as PTS. The phase factor $b_{i}$

*Corresponding author (email: zcwang@tsinghua.edu.cn) 
is introduced to multiply $\mathbf{x}_{\mathrm{ACO}}^{(i)}$ and adjust the phase of PTS, which is given by $b_{i}=\exp \left(j \frac{2 \pi}{W} l_{i}\right), l_{i}=$ $0,1, \ldots, W-1$. The time-domain signal $\mathbf{x}_{\mathrm{ACO}}(\mathbf{b})$ of modified ACO-OFDM is generated by combining the PTSs $\left(b_{i} \mathbf{x}_{\mathrm{ACO}}^{(i)}\right)$. For the sake of minimizing PAPR of the ACO-OFDM block, $\mathbf{b}$ is calculated with a low-complexity suboptimal algorithm proposed in [8]. In order to avoid data throughput loss induced by the transmission of side information, this letter exploits $N / 2$ unoccupied subcarriers to transmit the side information. In the proposed transmitter architecture, the integer indexes $\mathbf{l}$ are mapped to natural binary vector $\mathbf{c}_{l}=\left[c_{l, 0}, c_{l, 1}, \ldots, c_{l, V \log _{2} W-1}\right]$. All elements of $\mathbf{c}_{l}$ are mapped to BPSK constellations and assigned on the even non-zero subcarriers of PAM-DMT signal as $Y_{2 k+2}=\sqrt{E_{s, \mathrm{BPSK}}}\left(2 c_{l, k}-1\right)$, where $E_{s, \mathrm{BPSK}}$ denotes the average energy per BPSK symbol. The PAM-DMT signal carrying side information is obtained as

$$
\mathbf{Y}_{\mathrm{PAM}}=\left[0,0, j Y_{2}, 0, j Y_{4}, \ldots, j Y_{2 V \log _{2} W}, 0, \ldots, 0,-j Y_{2 V \log _{2} W}, 0,-j Y_{2 V \log _{2} W-2}, 0, \ldots,-j Y_{2}, 0\right] .
$$

The $N$-point IFFT block converts the side information block $\mathbf{Y}_{\text {PAM }}$ to PAM-DMT time-domain signal $\mathbf{y}_{\text {PAM }}$. The transmitted signal $\mathbf{x}$ could be directly generated by adding up the clipped data signal in ACO-OFDM and side information signal in PAM-DMT and used for modulating LEDs. In the proposed PAPR reduction scheme, the side information is modulated on the unoccupied even subcarriers and the total odd subcarriers are occupied by the data symbols, which helps the proposed scheme avoid the data throughput loss and achieve the same data throughput as conventional ACO-OFDM scheme.

It has been proved that the asymmetric clipping distortion of PAM-DMT signal only falls on the real part of even subcarriers, which has no effect on the data in ACO-OFDM [9]. Hence, the receiver can regenerate the ACO-OFDM and PAM-DMT signals to recover the phase factors and data sequentially [10]. In the proposed scheme, ACO-OFDM data and PAM-DMT side information are demodulated at the same signal-to-noise ratio (SNR) level in VLC channel. Since the gap between the demodulation SNR threshold of $M$-ary QAM constellation and BPSK is high, the allocated power of BPSK symbol could be decreased. In the proposed PTS-based ACO-OFDM scheme, power allocation ratio $\eta$ denoted as the ratio of $E_{s, \mathrm{QAM}}$ and $E_{s, \mathrm{BPSK}}$ is calculated as

$$
\eta=\frac{E_{s, \mathrm{QAM}}}{E_{s, \mathrm{BPSK}}}=\frac{2(M-1)}{3}\left[Q^{-1}\left(\frac{\sqrt{M} \log _{2} M}{4(\sqrt{M}-1)} P_{\mathrm{b}, \mathrm{QAM}}\right) / Q^{-1}\left(P_{\mathrm{b}, \mathrm{BPSK}}\right)\right]^{2}
$$

In order to restrict the performance loss induced by the error estimated phase factor at negligible level, the power allocation ratio $\eta$ is calculated at $P_{\mathrm{b}, \mathrm{BPSK}}=10^{-9}$ and $P_{\mathrm{b}, \mathrm{QAM}}=10^{-2}$. This high power allocation ratio $\eta$ mitigates the overall time-domain signal power $P_{E l e c \text {,all }}$ effectively, which is formulated as

$$
P_{\text {Elec }, \text { all }}=\left(1+\frac{4 V \log _{2} W}{N \eta}+2 \sqrt{\frac{4 V \log _{2} W}{\pi N \eta}}\right) P_{\text {Elec }, \mathrm{ACO}} .
$$

Simulation Results. Both the PAPR reduction and BER performance of the proposed PTS-based ACOOFDM scheme are demonstrated in comparison to its conventional ACO-OFDM counterpart depicted in Fig. 1. In the simulated VLC ACO-OFDM system, the subcarrier number $\mathrm{N}$ was set as 512 and the bandwidth $B$ was assumed as $200 \mathrm{MHz}$. 64QAM was adopted with the power allocation $\eta=7.1 \mathrm{~dB}$ calculated from (2) in the proposed ACO-OFDM. AWGN channel was assumed and the LED nonlinear transfer characteristic model given in [1] was applied. The data throughputs of the conventional and the proposed PTS-based ACO-OFDM schemes are both 300 Mbps.

Fig. 1-(a) illustrates the PAPR reduction performance of the PTS-based ACO-OFDM scheme. In the case of $V=4, W=16$, the proposed scheme offers the best PAPR reduction performance of around $5.1 \mathrm{~dB}$ at $\mathrm{CCDF}=10^{-4}$ in comparison to other PTS parameters. Therefore, we can conclude that larger available phase factor number $W$ could enhance the PAPR reduction performance when the total bit number of side information is kept unchanged. BER performance over AWGN channel with and without incorporating the LED nonlinear transfer characteristic is depicted in Fig. 1-(b). The proposed ACO-OFDM scheme suffers from performance losses of $0.45 \mathrm{~dB}$ and $0.54 \mathrm{~dB}$ at BER $=10^{-4}$ for the 

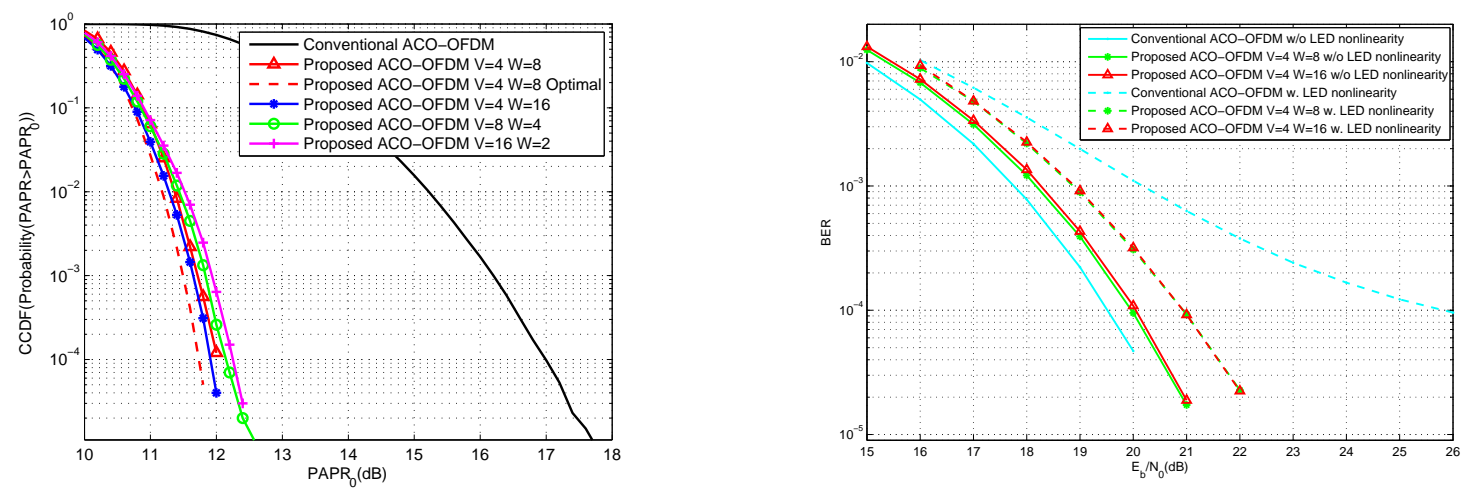

Figure 1 Performances of the PTS-based and the conventional ACO-OFDM. (a) PAPR CCDF curves; (b) BER curves.

side information number $V \log _{2} W$ of 12 and 16. The performance degradation mainly results from this slightly power increase induced by the transmitted side information signal, which is calculated as low as $0.43 \mathrm{~dB}$ and $0.51 \mathrm{~dB}$ according to (3). Besides that, Fig. 1-(b) shows that performance loss of the conventional ACO-OFDM scheme reaches a level of $6.3 \mathrm{~dB}$ at $\mathrm{BER}=10^{-4}$ when taking into account the LED nonlinear characteristic in VLC channel. In contrast to the convention ACO-OFDM, the proposed scheme achieves a significant performance gain of $5.0 \mathrm{~dB}$ at $\mathrm{BER}=10^{-4}$.

Conclusions. In this letter, we propose a PTS-based PAPR reduction scheme for ACO-OFDM in VLC systems in order to mitigate high PAPR issue of ACO-OFDM. The proposed scheme avoids the data throughput loss by transmitting the side information on unoccupied subcarriers and an effective power allocation strategy is derived to enhance the performance. Simulation results demonstrate that the PTSbased PAPR reduction scheme for ACO-OFDM achieves a tremendous PAPR reduction and mitigates the negative effect of the LED nonlinear distortion significantly.

\section{Acknowledgements}

This work was supported by National Key Basic Research Program of China (Grant No. 2013CB329203), National Nature Science Foundation of China (Grant No. 61271266), Shenzhen Visible Light Communication System Key Laboratory (ZDSYS20140512114229398), Shenzhen Wireless over Visible Light Communication Technology Engineering Laboratory(SDRC[2012]No.1440) and Shenzhen Peacock Plan (No. 1108170036003286).

\section{References}

1 Dimitrov S, Haas H. Information rate of OFDM-based optical wireless communication systems with nonlinear distortion. IEEE/OSA J Lightw Technol, 2013, 31: 918-929

2 Armstrong J, Lowery A J. Power efficient optical OFDM. Electron Lett, 2006, 42 370-372

3 Kong L, Shen $\mathrm{H}, \mathrm{Xu} \mathrm{W}$, et al. On capacity-achieving transmissions for multiuser VLC systems using LED array. to appear in Sci China Inf Sci

4 Popoola W O, Ghassemlooy Z, Stewart B G. Pilot-assisted PAPR reduction technique for optical OFDM communication systems. IEEE/OSA J Lightw Technol, 2014, 32 1374-1382

5 Yu Z, Baxley R J, Zhou G T. Iterative clipping for PAPR reduction in visible light OFDM communication. In: Proceeding of the IEEE MILCOM, Baltimore, 2014. 1681-1686

$6 \mathrm{Xu} \mathrm{W}$, Wu M, Zhang H, et al. ACO-OFDM-specified recoverable upper clipping with efficient detection for optical wireless communications. IEEE Photon J, 2014, 6

7 Ranjha B, Kavehrad M. Precoding techniques for PAPR reduction in asymmetrically clipped OFDM based optical wireless system. In: Proceeding of the SPIE, 2013. 86450R

8 Han S H, Lee J H. PAPR reduction of OFDM signals using a reduced complexity PTS technique. IEEE Sig Proc Lett, 2004, 11: 887-890

9 Wang Q, Wang Z C, Dai L L. Asymmetrical hybrid optical OFDM for visible light communications with dimming control. IEEE Photon Technol Lett, 2015, 27: 974-977

10 Wang Q, Wang Z C, Dai L L. Iterative receiver for hybrid asymmetrically clipped optical OFDM. IEEE/OSA J Lightw Technol, 2014, 32: 3869-3875 